\title{
Commentary
}

\section{Broadening our understanding of Canada's epidemics of pharmaceutical and contaminated street drug opioid-related overdoses}

\author{
Robert Strang, MD, MHSc, FRCPC
}

It is my privilege to introduce this second special issue of Health Promotion and Chronic Disease Prevention in Canada: Research, Policy and Practice focussed on Canada's current opioid crisis. As Nova Scotia's Chief Medical Officer of Health, I deal with a broad range of public health issues. The opioid crisis is one of the most challenging, given its magnitude, reach and complex nature. The death toll is sobering: in 2017 alone, almost 4000 persons lost their lives to apparent opioidrelated overdoses in Canada, and this figure may rise as more data become available. ${ }^{1}$ Every part of the country and all sectors of Canadian society are affected. The majority of these deaths are occurring in men $(78 \%)$ and among individuals aged 30 to 39 years (28\%). ${ }^{1}$ The evidence suggests that apparent opioidrelated overdoses may now be the leading cause of death among men aged 30 to $39 .^{2}$ One of the reasons this opioid crisis is so complex is that it is actually made up of two linked epidemics: an epidemic of harms, including overdoses, from pharmaceutical opioids, and an epidemic of overdoses from contaminated street drugs.

When federal, provincial and territorial governments established the Special Advisory Committee on the Epidemic of Opioid Overdoses (SAC), which I co-chair with my federal counterpart, Dr. Theresa Tam, Canada's Chief Public Health Officer, we did not even know how many Canadians were dying every year from opioid overdoses. Setting up a national surveillance system to monitor apparent opioid-related deaths was itself a significant accomplishment, requiring close cooperation between the sectors of justice, public/community safety and health in all 13 provinces and territories, as well as the federal government. While this new national cross-sector collaboration was imperative to allow us to quantify the critical outcome of opioidrelated overdose deaths, it is just one of many partnerships required to effectively address this crisis. An effective and coordinated response depends on a wide range of timely evidence, which has necessitated collaboration between the public health sector and non-traditional government counterparts in justice, public/community safety and border security, as well as first responders, primary care providers, harm reductions agencies and persons with lived experiences, among others. While we now have data on and some understanding of proximate determinants-for example, we know that in 2017 threequarters $(72 \%)$ of apparent opioid-related deaths involved fentanyl or fentanyl analogues, and that the majority (71\%) of accidental deaths also involved one or more non-opioid substances ${ }^{1}$ - there is a tremendous amount to be done to describe and quantify the complex web of upstream causes of the linked epidemics of pharmaceutical opioid and contaminated street drug overdoses.

The articles in this issue must be understood in this larger context: we know more than we did in December 2016, when the SAC was established, but we need to know much more still to significantly reduce the number of Canadians dying from pharmaceutical and street drug opioid-related overdoses. Collectively, these articles increase our understanding of Canada's opioid crisis and help to inform our public health interventions.
The first three articles examine several current surveillance initiatives that provide insight on different facets of the crisis from a national perspective. Abdesselam ${ }^{3}$ highlights initiatives undertaken by the Government of Canada and other national and provincial and territorial partners to monitor the opioid crisis, and makes the case for novel surveillance approaches to improve the collection, analysis and harmonization of drug-related data, such as the development of a national drug observatory. Do and colleagues ${ }^{4}$ use national data on opioid toxicity-related emergency department visits, from the Canadian Injury Reporting and Prevention Program (eCHIRPP), a network of 6 general and 11 pediatric hospitals from across the country, to examine associated context and circumstances surrounding these visits. Their study provides preliminary insights into the influence of proximal and distal/upstream factors associated with opioid use, highlighting the importance of mental health as an apparent contributing factor. Do ${ }^{5}$ also teamed up with the City of Ottawa Paramedic Service to provide a "proof of concept" for national surveillance of Emergency Medical Services (EMS) data on suspected overdoses occurring prior to admission to a health care service. Their proof of concept demonstrates that, when available, paramedic data can serve as a rich, timely source of information.

The following three articles provide insights on opportunities for interventions, from addressing upstream factors such as access to supply and mental health, to engaging downstream health systems to monitor opioid-related harms 
and health outcomes. In British Columbia, Otterstatter ${ }^{6}$ describes patterns of health care utilization among people who overdosed from illegal street drugs, using the BC provincial overdose cohort. ${ }^{6}$ His finding, that overdose cases have higher rates of health service use, suggests that there are opportunities within the health care system, missed and otherwise, to provide interventions and to take preventative action. In Nova Scotia, Schleihauf ${ }^{7}$ and team describe the impact of changes to opioid prescribing practices on opioidrelated death rates. They found that while opioid-related mortality rates remained more or less constant between 2011 and 2017, the number of prescription opioid morphine units distributed by pharmacists decreased, while the proportion of deaths attributed to nonpharmaceutical opioids increased following recent changes to prescribing guidelines. This finding highlights the importance of monitoring both prescriptions and the supply of street drugs, in order to determine the impact of changes in prescribing practices on substance use patterns. In Alberta, $\mathrm{Chan}^{8}$ and colleagues examined opioid toxicity suicide trends from 2000 to 2016, and their burden relative to other means of suicide. They did not observe similar trends between opioid-related intentional overdoses (suicide) and illegal fentanyl-driven unintentional overdoses (accidental), suggesting that individuals who die by opioid toxicity suicide are different from those who die from an accidental overdose, and that public health interventions must therefore be tailored to each group.

Over the past two years, governments at the federal, provincial and territorial levels have mobilized technical and financial resources to respond to this crisis. In line with the federal government's four-pillared approach to the opioid crisis ${ }^{9}$ (prevention, treatment, harm reduction and enforcement), many provinces and territories have put in place coordinated action plans and strategies to respond to the crisis. Public health surveillance has been, and will continue to be, critical to informing effective, coordinated responses. However, it will need to expand beyond monitoring and tracking health outcomes and pharmaceutical and street drug supply and use, to exploring the deeper social and structural determinants of health, i.e., the "causes of the causes." 10 Understanding upstream causative factors will require engaging a broader range of sectors to be more proactive in developing and targeting interventions and prevention strategies.

The work that has been done in the area of surveillance continues to provide evidence needed to inform this crisis and the move further upstream is groundbreaking. The lessons learned will serve us well as we look at substance use more broadly, including the impact of the upcoming cannabis legislation.

I would like to take this opportunity to commend all those working in public health and across the partnering disciplines for your collaborative and determined work collecting and analyzing the evidence, informing and supporting the work to address these linked epidemics.

\section{Dr. Robert Strang \\ Chief Medical Officer of Health Nova Scotia Department of Health and Wellness}

\section{References}

1. Special Advisory Committee on the Epidemic of Opioid Overdoses. National report: apparent opioid-related deaths in Canada (January 2016 to December 2017) [Internet]. Ottawa (ON): Public Health Agency of Canada; 2017 [cited 2018 July]. Available from: https:// www.canada.ca/en/public-health /services/publications/healthy-living /national-report-apparent-opioid-related -deaths-released-june-2018.html

2. Chief Public Health Officer announcement at joint Public Health Agency of Canada and Canadian Institutes for Health Research Technical Briefing, June 19, 2018, Ottawa, Ontario, Canada.

3. Abdesselam K, Dann M J, Alwis R, et al. At-a-glance - Opioid surveillance: monitoring and responding to the evolving crisis. Health Promot Chronic Dis Prev Can. 2018;38(9):312-6.

4. Do $M$, Chang V, Tibebu S, et al. Sentinel surveillance of suspected opioid-related poisonings and injuries: trends and context derived from the electronic Canadian Hospitals Injury Reporting and Prevention Program, March 2011 to June 2017. Health Promot Chronic Dis Prev Can. 2018;38(9):317-27.
5. Do M, Furlong G, Leyenaar $M$, et al. At-a-glance - What can paramedic data tell us about the opioid crisis in Canada? Health Promot Chronic Dis Prev Can. 2018;38(9):339-42.

6. Otterstatter M, Crabtree A, Dobrer S, et al. Patterns of health care utilization among people who overdosed from illegal drugs: a descriptive analysis using the $\mathrm{BC}$ Provincial Overdose Cohort. Health Promot Chronic Dis Prev Can. 2018;38(9):328-33.

7. Schleihauf E, Crabtree K, Dohoo C, et al. At-a-glance - Concurrent monitoring of opioid prescribing practices and opioid-related deaths: the context in Nova Scotia, Canada. Health Promot Chronic Dis Prev Can. 2018; 38(9):334-8.

8. Chan E, McDonald B, Brooks-Lim E, et al. At-a-glance - The role of opioid toxicity in suicide deaths in Alberta, 2000 to 2016. Health Promot Chronic Dis Prev Can. 2018;38(9):343-7.

9. Government of Canada. Government of Canada actions on opioids: 2016 and 2017 [Internet]. Ottawa (ON): Government of Canada; 2017 [cited 2018 July]. Available from: https:// www.canada.ca/en/health-canada /services/publications/healthy-living /actions-opioids-2016-2017.html

10. Marmot M. Social determinants of health inequalities. Lancet. 2005; 365(9464):1099-104. 